\title{
OPEN Genome and transcriptome profiling of spontaneous preterm birth phenotypes
}

\author{
Juhi K. Gupta ${ }^{1,2 \bowtie}$, Angharad Care2 ${ }^{2}$ Laura Goodfellow ${ }^{2}$, Zarko Alfirevic ${ }^{2}$, \\ Bertram Müller-Myhsok ${ }^{1,3}$ \& Ana Alfirevic ${ }^{1,2}$
}

Preterm birth (PTB) occurs before 37 weeks of gestation. Risk factors include genetics and infection/ inflammation. Different mechanisms have been reported for spontaneous preterm birth (SPTB) and preterm birth following preterm premature rupture of membranes (PPROM). This study aimed to identify early pregnancy biomarkers of SPTB and PPROM from the maternal genome and transcriptome. Pregnant women were recruited at the Liverpool Women's Hospital. Pregnancy outcomes were categorised as SPTB, PPROM ( $\leqslant 34$ weeks gestation, $n=53$ ), high-risk term (HTERM, $\geq 37$ weeks, $n=126$ ) or low-risk (no history of SPTB/PPROM) term (LTERM, $\geq 39$ weeks, $n=188$ ). Blood samples were collected at 16 and 20 weeks gestation from which, genome (UK Biobank Axiom array) and transcriptome (Clariom D Human assay) data were acquired. PLINK and R were used to perform genetic association and differential expression analyses and expression quantitative trait loci (eOTL) mapping. Several significant molecular signatures were identified across the analyses in preterm cases. Genome-wide significant SNP rs14675645 (ASTN1) was associated with SPTB whereas microRNA-142 transcript and PPARG1-FOXP3 gene set were associated with PPROM at week 20 of gestation and is related to inflammation and immune response. This study has determined genomic and transcriptomic candidate biomarkers of SPTB and PPROM that require validation in diverse populations.

Preterm birth (PTB) occurs when an infant is born prior to 37 completed weeks of gestation and is a major public health issue. PTB is a multifactorial condition and is associated with a number of poor health outcomes in infants including cerebral palsy, problems with vision and hearing, poor motor skills, asthma, autism and increased metabolic and cardiovascular risks to health ${ }^{1,2}$. Spontaneous PTB (sPTB) accounts for approximately two-thirds of all PTB and describes women that labour early, often for no clear reason. The other one-third are healthcare-provider initiated, usually in response to severe maternal or fetal disease (e.g. pre-eclampsia or severe intrauterine growth restriction) and will not be considered further in this study. A higher rate of infant morbidity is associated with $\leq 34$ weeks of gestation and therefore this was applied as the threshold for sPTB in this study ${ }^{3}$.

Studies have shown that a familial hereditary element increases the risk of early labour. Women who were born preterm were more likely have a preterm delivery, and a women who had an obstetric history of sPTB is more likely to have a subsequent $\mathrm{sPTB}^{4-7}$. Both environmental factors (such as infection or lifestyle choices) and genetic factors are risks also associated with $\mathrm{sPTB}^{8,9}$. The role of infection pathway and associated genes, interleukins or tumour necrosis factors (TNF) has been reported in many studies, suggesting that the maternal genome should be screened for potential genetic biomarkers ${ }^{10-14}$. A large PTB GWAS study by Zhang et al. ${ }^{15}$, consisting of over 43,000 women, identified EBF1 variant (Early B-cell factor 1, a transcription factor) associated with an increased risk of PTB further supporting a genetic cause involving immune response/inflammatory pathways.

Two clinical phenotypes of sPTB are (1) spontaneous preterm birth (SPTB) following the spontaneous onset of labour and (2) preterm premature rupture of membranes (PPROM) where the amniotic membrane spontaneously breaks and increases the risk of infection or preterm labour at a later date ${ }^{16}$. Despite obvious clinical differences, these two subgroups are often not differentiated in research studies when determining biomarkers for risk prediction, despite a universal acceptance that SPTB has multiple causes ${ }^{17}$. However, Capece et al. ${ }^{18}$ conducted

\footnotetext{
${ }^{1}$ Wolfson Centre for Personalised Medicine, Department of Pharmacology and Therapeutics, Institute of Systems, Molecular and Integrative Biology, University of Liverpool, Block A: Waterhouse Buildings, 1-5 Brownlow Street, Liverpool L69 3GL, UK. ${ }^{2}$ Harris-Wellbeing Research Centre, University Department, Liverpool Women's Hospital, Liverpool L8 7SS, UK. ${ }^{3}$ Max Planck Institute of Psychiatry, 80804 Munich, Germany. ${ }^{\boxplus}$ email: J.Gupta@ liverpool.ac.uk
} 


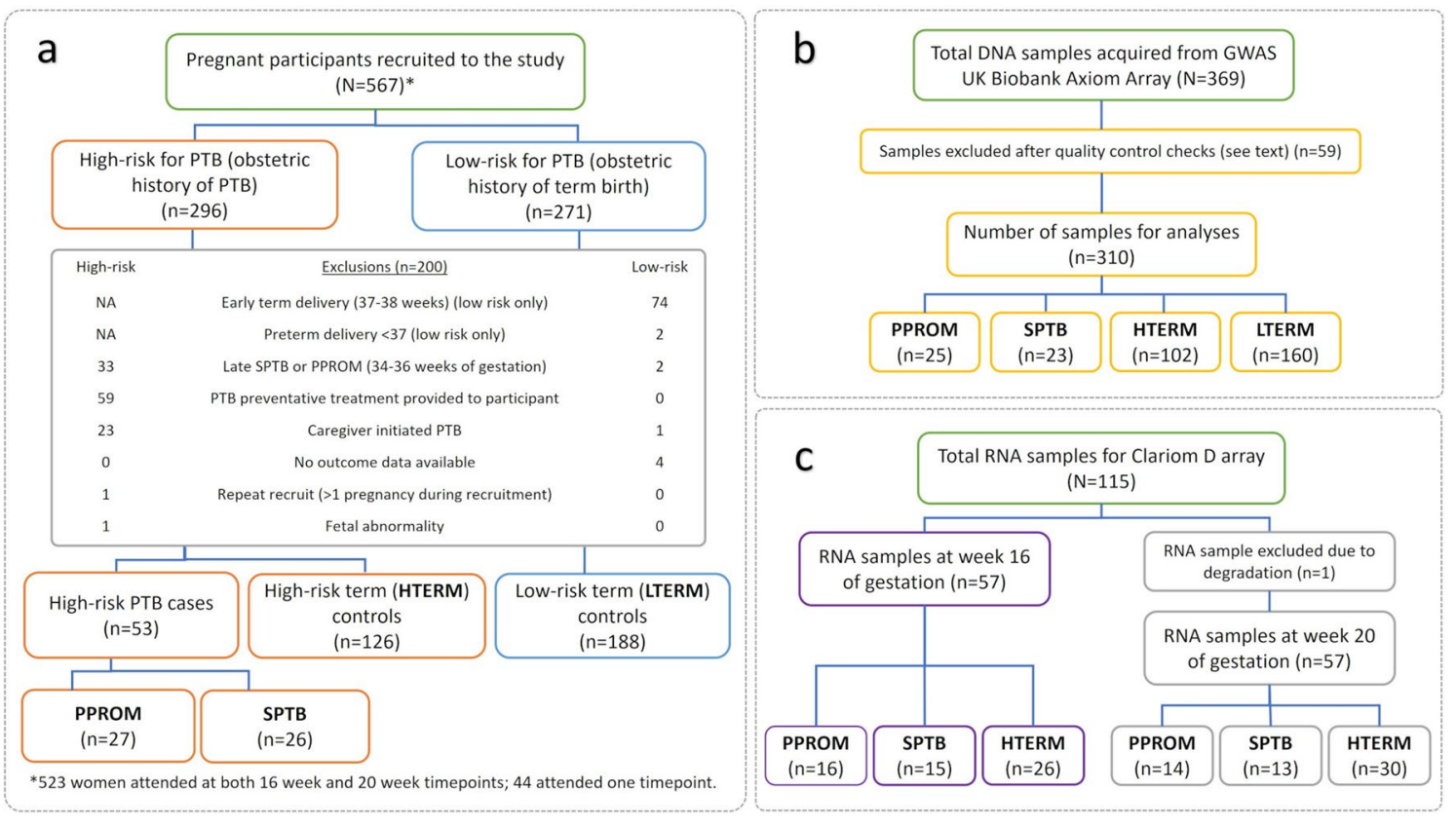

Figure 1. Singleton pregnant participants recruited to the Liverpool preterm birth study cohort. (a) Final number of women included in the analyses. (b) DNA was extracted from whole blood collected from the participants and genomic profiling (GWAS) was conducted on the Biobank Axiom ${ }^{\mathrm{Tw}}$ array. (c) RNA extracted from whole blood collected from participants at 16 and/or 20 weeks of gestation was processed on the Clariom D array. GWAS genome-wide association study, HTERM high-risk term births, LTERM low-risk term births, PPROM preterm premature rupture of membranes, $P T B$ preterm birth, SPTB spontaneous preterm birth.

pathway analysis of PTB genetic studies and proposed the role of immune and hormonal regulation in SPTB, versus the role of hematologic disorder, collagen metabolism, matrix degradation and local inflammation in PPROM. Differences in response to PTB preventative treatments, of women with a history of either PPROM or SPTB, in a subsequent pregnancy was described by Care et al. ${ }^{19}$, suggesting these women are different, and may have unique genetics influencing their response to known clinical treatments.

Recent PTB studies have identified the association of PTB with maternal microRNAs ${ }^{20-24}$. Short, non-coding microRNAs are key post-transcriptional regulators, regulating gene expression by destabilising mRNA and thereby repressing protein production ${ }^{25,26}$. Exploring expression quantitative trait loci (eQTL) can determine associations between SNPs and transcripts to gain functional insights on candidate biomarkers using linear models ${ }^{27}$.

No study has attempted to link genetic inheritance with gene expression in women with SPTB and PPROM. We have acquired and analysed genome-wide SNP data and transcriptome gene expression data from the same cohort of women collected simultaneously from maternal blood during pregnancy to perform eQTL mapping to determine potential biomarkers of spontaneous preterm birth.

To our knowledge, this is the first investigation to explore both genome-wide and transcriptome-wide profiles in prospectively collected, mid-trimester maternal blood samples (from a well-defined cohort, using a PTB cut-off of $\leq 34$ weeks gestation), to determine biomarkers of spontaneous preterm birth phenotypes PPROM and SPTB.

\section{Results}

Figure 1a summarises the number of women recruited to the study. A total of 310 DNA samples were included in GWAS analyses (Fig. 1b) and 114 RNA samples in gene expression analysis (Fig. 1c).

GWAS SNP associations. GWAS analyses were conducted on the PTB phenotypes (cases), (1) SPTB and (2) PPROM against the term birth groups, (3) LTERM (recurrent term births only) and (4) HTERM (previous PTB with subsequent term birth).

PPROM versus LTERM. PPROM versus LTERM analysis resulted in two genome-wide significant SNPs at chromosome 4, rs187066376 $(\mathrm{p}=5.71 \mathrm{e}-09)$ and $\mathrm{rs} 151199874(\mathrm{p}=5.11 \mathrm{e}-08)$ both in non-coding region, LOC105377408 (Fig. 2a). SNP towers at chromosome 10 (rs34638554) and 12 (rs77423197) were further identified in non-coding regions (Fig. 2b).

SPTB versus HTERM. SPTB versus HTERM association analysis yielded a genome-wide significant SNP on chromosome 1 ( $r s 146756455, \mathrm{p}=3.18 \mathrm{E}-08$ ) and a suggestive SNP signal, close to genome-wide significance threshold, on chromosome 4 (Fig. 3a). The genome-wide significant SNP, rs146756455, is an intronic variant of 

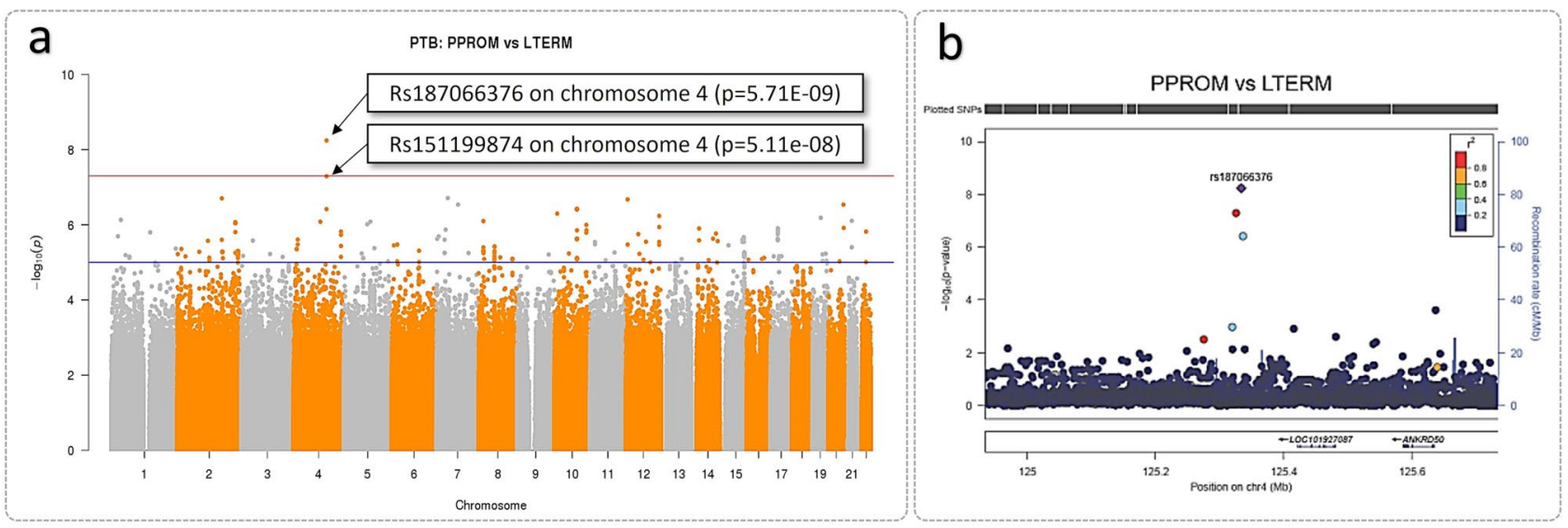

Figure 2. Genome-wide significant SNP identified from PPROM cases and LTERM controls GWAS analysis. (a) Manhattan plot of PPROM $(n=25) \leq 34$ weeks gestation and LTERM $(n=160) \geq 39$ weeks gestation GWAS analysis using Frequentist association test. Multi-dimensional scaling components 1 to 6 of the cohort were included as covariates. The upper red horizontal line displays the genome-wide significance threshold $\left(\mathrm{p}<5 \times 10^{-8}\right)$ and the lower blue horizontal line represents an arbitrary suggestive threshold $\left(\mathrm{p}<1 \times 10^{-5}\right)$. One SNP (rs187066376) exceeded genome-wide significance in this analysis on chromosome 4 ( $\mathrm{p}=5.71 \mathrm{E}-09)$. Figure generated using R package 'qqman'28. (b) Regional plot of rs187066376 signal. Two SNPs are shown in linkage disequilibrium in red and blue. Figure produced using LocusZoom ${ }^{29}$.

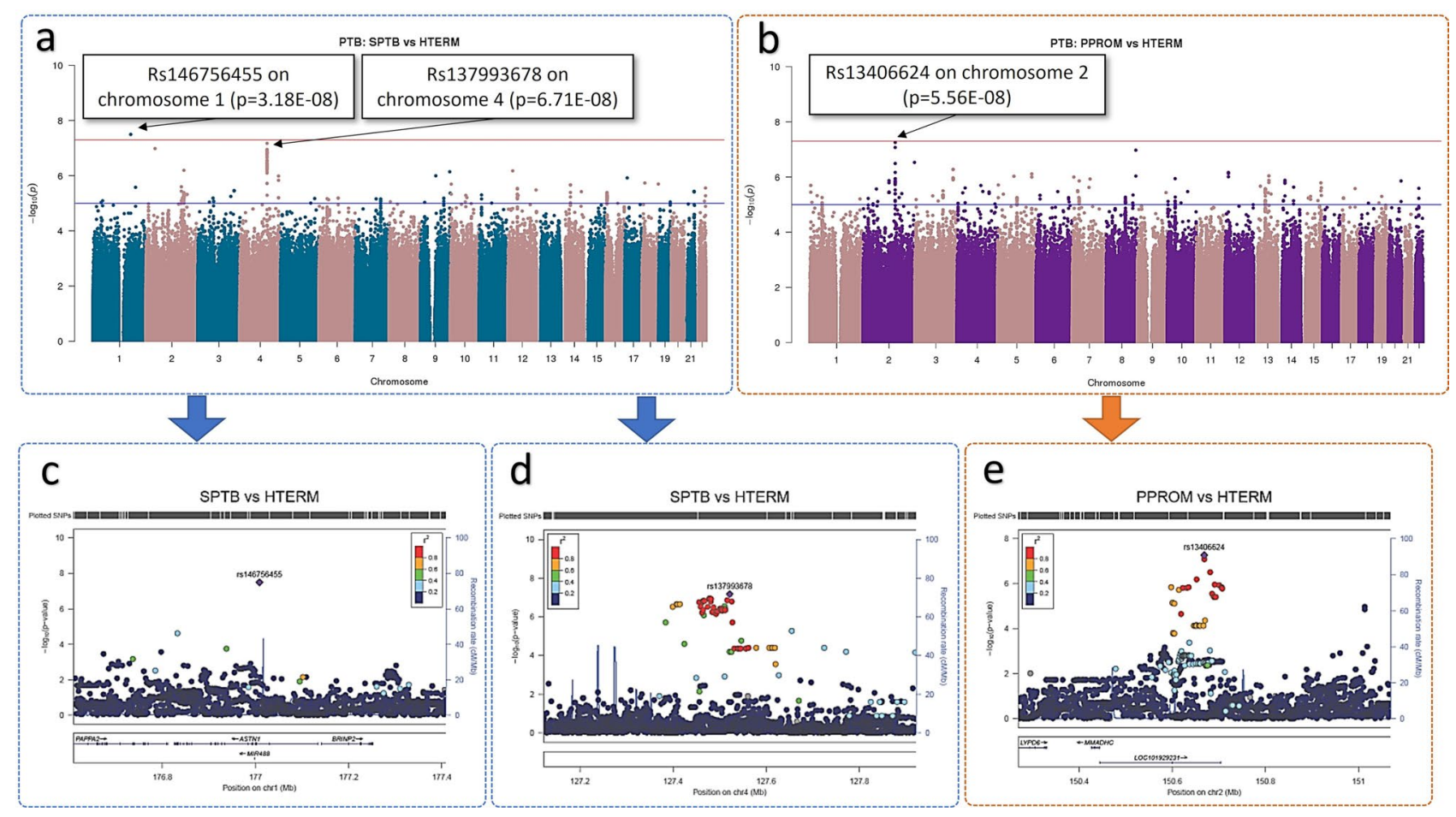

Figure 3. Manhattan plots of preterm versus term births GWAS analyses using Frequentist association test. (a) SPTB $(n=23) \leq 34$ weeks gestation and HTERM $(n=102) \geq 37$ weeks gestation and (b) PPROM $(n=25) \leq 34$ weeks gestation and HTERM $(n=102) \geq 37$ weeks gestation. Multi-dimensional scaling components 1 to 6 of the cohort were included as covariates. The upper red horizontal line displays the genome-wide significance threshold $\left(\mathrm{p}<5 \times 10^{-8}\right)$ and the lower blue horizontal line represents an arbitrary suggestive threshold $\left(\mathrm{p}<1 \times 10^{-5}\right)$. Manhattan plots were generated using R package 'qqman ${ }^{28}$. Regional plots: (c) genome-wide significant SNP (rs146756455) on chromosome 1 was identified as an intron variant in the gene, ASTN1 ( $\mathrm{p}=3.18 \mathrm{E}-08$ ) (SPTB versus HTERM); (d) rs137993678 approaching genome-wide significance on chromosome $4(\mathrm{p}=6.71 \mathrm{E}-08)$ (SPTB versus HTERM). Several SNPs are in linkage disequilibrium; (e) rs13406624 on chromosome 2 was identified as a non-coding region, LINC01931 (or MMADHC-DT) $(\mathrm{p}=5.56 \mathrm{E}-08)$ (PPROM versus HTERM). Regional plots were produced using LocusZoom ${ }^{29}$. 

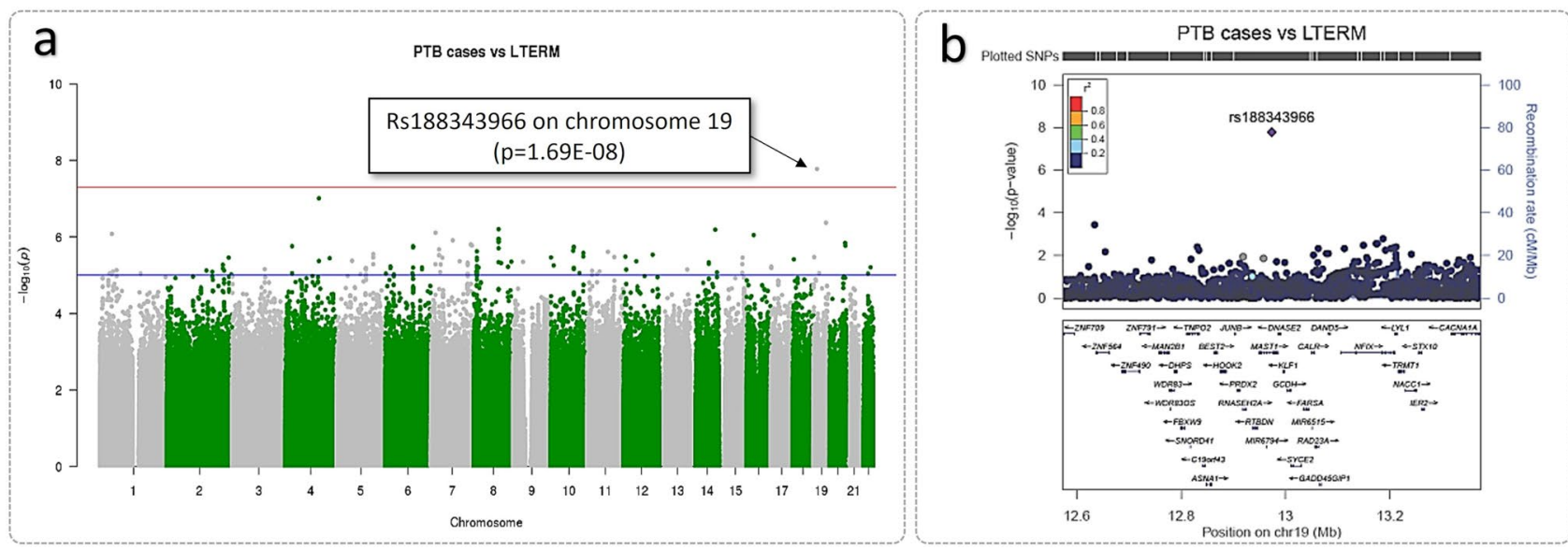

Figure 4. Genome-wide significant SNP identified from PTB cases and LTERM controls GWAS analysis. (a) Manhattan plot of all PTB cases $(n=48) \leq 34$ weeks gestation and LTERM $(n=160) \geq 39$ weeks gestation GWAS analysis using Frequentist association test. Multi-dimensional scaling components 1 to 6 of the cohort were included as covariates. The upper red horizontal line displays the genome-wide significance threshold $\left(\mathrm{p}<5 \times 10^{-8}\right)$ and the lower blue horizontal line represents an arbitrary suggestive threshold $\left(\mathrm{p}<1 \times 10^{-5}\right)$. One SNP (rs188343966) exceeded genome-wide significance in this analysis on chromosome 19 ( $\mathrm{p}=1.69 \mathrm{E}-08)$. Figure generated using R package 'qqman'28. (b) Regional plot of rs188343966 signal. Figure produced using LocusZoom ${ }^{29}$.

ASTN1 ( $\mathrm{p}=3.18 \mathrm{E}-08)$, which encodes astrotactin 1 (Fig. 3c). The association signal approaching genome-wide significance threshold at chromosome 4 (rs137993678) was not in a gene coding region (Fig. $3 \mathrm{~d}$ ).

PPROM versus HTERM. No genome-significant SNPs resulted from the PPROM versus HTERM GWAS analysis. The SNP signal rs 13406624 at chromosome $2(\mathrm{p}=5.56 \mathrm{E}-08)$, approaching genome-wide significance, was in a non-coding region (Fig. 3b). Multiple SNPs were in the linkage disequilibrium (LD) with rs13406624 at chromosome 2 in non-coding RNA, LINC01931 (or MMADHC divergent transcript) (Fig. 3e).

SPTB versus LTERM. Association analysis between SPTB and LTERM births yielded no genome-wide significant SNPs, however multiple signals were obtained (Supplementary Fig. S1, see Supplementary File 1). An association signal at chromosome 10 (rs9424165) obtained the lowest p-value is an intronic variant in CAMK1D (calcium/calmodulin-dependent protein kinase ID). Chromosome $1 \mathrm{rs} 2092868$ is an intron variant in NFIA (Nuclear Factor I A) region, chromosome 2 signal (rs78202288) is a MYO3B (myosin IIIB) intron variant, chromosome 3 (rs61796814) signal is not in a coding region and chromosome 17 signal (rs150140114) is an intron variant in AKAP10 (A-kinase anchor protein 10) region.

LTERM versus SPTB cases. Analysis of SPTB cases and LTERM highlighted a genome-wide significant SNP, rs188343966 ( $\mathrm{p}=1.69 \mathrm{E}-08$, chr19:12973701), which is an intron variant in gene MAST1 (Microtubule Associated Serine/Threonine Kinase 1) (Fig. 4a,b). No SNPs were identified in linkage disequilibrium with MAST1 (rs188343966) (Fig. 4b).

HTERM versus sPTB cases. No genome-wide significant SNPs were identified from the sPTB cases versus HTERM GWAS analysis (Supplementary Fig. S2). The lowest p-value signal on chromosome 16 (rs59159780, $\mathrm{p}=4.14 \mathrm{E}-07)$ is not within a gene region (Supplementary Fig. S2).

Differential gene expression analysis at week 16 of gestation. A total of 30,120 differentially expressed transcripts were identified at week 16 of gestation $(\mathrm{p}<0.05)$, when analysing gene expression across SPTB versus HTERM, PPROM versus HTERM and SPTB versus PPROM. However none were significant at FDR $\mathrm{p}<0.05$ and did not reach log fold change threshold of $<1.5$ or $>1.5$.

Differential gene expression analysis at week 20 of gestation. At week 20 of gestation, 147 differentially expressed genes (DEGs) (49 upregulated and 98 downregulated) were significant at FDR $\mathrm{p}<0.05$ for the PPROM versus HTERM analysis (Fig. 5a). Of these significant DEGs, none reached $>1.5$ or $<-1.5$ log fold change (Fig. 5b; Supplementary File 2) (Fig. 5c). An unknown transcript (Probe ID: TC0300009931.hg.1), with no annotation available, yielded the lowest $\mathrm{p}$-value $(\mathrm{p}=1.19 \mathrm{E}-07, \mathrm{FDR} \mathrm{p}=0.02)$. Of the transcripts with annotations available, the top significant findings (FDR $\mathrm{p}<0.03$ ) are highlighted in Fig. $5 \mathrm{c}$ heatmap and summarised in Table 1. Full results from this analysis can be found in Supplementary File 2. No significant results were determined for SPTB versus HTERM or SPTB versus PPROM. 


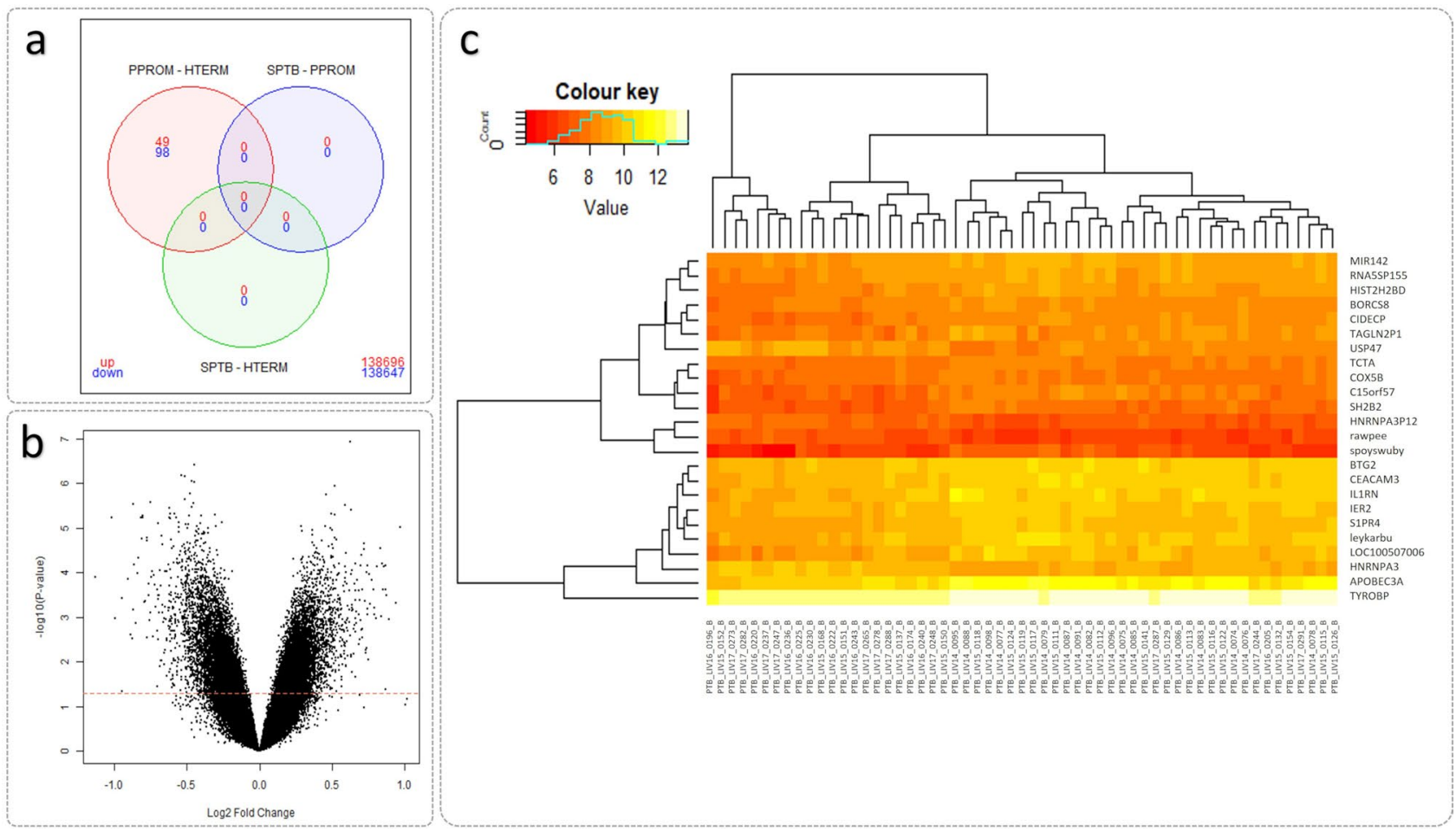

Figure 5. Significant DEGs identified from PPROM versus HTERM analysis at week 20 gestation of 57 maternal samples. (a) Venn diagram of 147 DEGs (49 upregulated and 98 downregulated) were significant at FDR $\mathrm{p}<0.05$. (b) Volcano plot of DEGs based on nominal $\mathrm{p}<0.05$. (c) Heatmap outlining 24 DEGs (with annotations available) that reached FDR $\mathrm{p}<0.03$. These figures were produced suing 'limma' $\mathrm{R}$ package ${ }^{30}$.

\begin{tabular}{|c|c|c|c|c|c|}
\hline ID & Symbol & Genename & $\log \mathrm{FC}^{*}$ & p & FDR $\mathbf{P}^{\dagger}$ \\
\hline NR_029683 & MIR142 & MicroRNA 142 & -0.513 & $6.79 \mathrm{E}-07$ & 0.022 \\
\hline NM_001862 & COX5B & Cytochrome $\mathrm{c}$ oxidase subunit $\mathrm{Vb}$ & -0.451 & $9.51 \mathrm{E}-07$ & 0.022 \\
\hline ENST00000437410 & HNRNPA3P12 & $\begin{array}{l}\text { Heterogeneous nuclear ribonucleoprotein A3 pseudo- } \\
\text { gene } 12\end{array}$ & 0.518 & $1.16 \mathrm{E}-06$ & 0.023 \\
\hline NM_194247 & HNRNPA3 & Heterogeneous nuclear ribonucleoprotein A3 & 0.458 & $1.79 \mathrm{E}-06$ & 0.028 \\
\hline NM_003775 & S1PR4 & Sphingosine-1-phosphate receptor 4 & -0.513 & $2.28 \mathrm{E}-06$ & 0.028 \\
\hline NM_004907 & IER2 & Immediate early response 2 & -0.453 & $2.73 \mathrm{E}-06$ & 0.028 \\
\hline NM_001080791 & C15orf57 & Chromosome 15 open reading frame 57 & -0.867 & $2.87 \mathrm{E}-06$ & 0.028 \\
\hline NM_001173514 & TYROBP & TYRO protein tyrosine kinase binding protein & -0.466 & $3.40 \mathrm{E}-06$ & 0.028 \\
\hline ENST00000411154 & RNA5SP155 & RNA, 5S ribosomal pseudogene 155 & -0.622 & $3.56 \mathrm{E}-06$ & 0.028 \\
\hline NM_001282659 & USP47 & Ubiquitin specific peptidase 47 & 0.634 & $4.02 \mathrm{E}-06$ & 0.028 \\
\hline NR_002786 & CIDECP & Cell death-inducing DFFA-like effector c pseudogene & -0.583 & $4.78 \mathrm{E}-06$ & 0.028 \\
\hline NM_020979 & $\mathrm{SH} 2 \mathrm{~B} 2$ & SH2B adaptor protein 2 & -0.613 & $4.85 \mathrm{E}-06$ & 0.028 \\
\hline NM_022171 & TCTA & T-cell leukaemia translocation altered & -0.305 & $4.93 \mathrm{E}-06$ & 0.028 \\
\hline NM_006763 & BTG2 & BTG family, member 2 & -0.482 & $5.22 \mathrm{E}-06$ & 0.028 \\
\hline NM_001145783 & BORCS8 & BLOC-1 related complex subunit 8 & -0.400 & $5.31 \mathrm{E}-06$ & 0.028 \\
\hline OTTHUMT00000321237 & APOBEC3A & $\begin{array}{l}\text { Apolipoprotein B mRNA editing enzyme, catalytic } \\
\text { polypeptide-like 3A }\end{array}$ & -0.807 & $5.33 \mathrm{E}-06$ & 0.028 \\
\hline OTTHUMT00000380415 & TAGLN2P1 & Transgelin 2 pseudogene 1 & -0.623 & $5.90 \mathrm{E}-06$ & 0.028 \\
\hline NM_001277163 & CEACAM3 & $\begin{array}{l}\text { Carcinoembryonic antigen-related cell adhesion } \\
\text { molecule } 3\end{array}$ & -0.575 & $5.93 \mathrm{E}-06$ & 0.028 \\
\hline NM_000577 & IL1RN & Interleukin 1 receptor antagonist & -0.816 & $5.99 \mathrm{E}-06$ & 0.028 \\
\hline NR_120420 & LOC100507006 & Uncharacterized LOC100507006 & -0.804 & $5.99 \mathrm{E}-06$ & 0.028 \\
\hline OTTHUMT00000087130 & HIST2H2BD & Histone cluster 2, H2bd (pseudogene) & -0.617 & $6.28 \mathrm{E}-06$ & 0.028 \\
\hline
\end{tabular}

Table 1. Top 21 differentially expressed genes detected from PPROM versus HTERM expression analysis at week 20 of gestation. ${ }^{\star} \log \mathrm{FC}=\log 2$ (fold change). ${ }^{\dagger}$ Significant at FDR $\mathrm{p}<0.03$, not including 3 unspliced transcripts. 
Lysophosphatidic acid receptor pathway identified. Of the 147 significant DEGs detected for week 20 PPROM versus HTERM comparison, 119 were associated with gene symbols and uploaded on Reactome for pathway analysis. Lysosphingolipid and Lysophosphatidic acid (LPA) receptors pathway was determined as significant at $\mathrm{p}<0.05(\mathrm{p}=0.0096)$, however this was not significant at FDR $\mathrm{p}<0.05$.

Gene set enrichment analysis at week 16 of gestation. Several significant gene sets from FUMA $($ FDR $\mathrm{p}<0.05)$ were obtained: SPTB versus HTERM $(\mathrm{n}=1864)$, SPTB versus PPROM $(\mathrm{n}=372)$ and PPROM versus HTERM $(n=3148)$. Many of these were reported as 'chemical and genetic perturbations'.

Gene set enrichment analysis at week 20 of gestation. Similar significant gene sets as week 16 of gestation were also identified at week 20 (FDR p <0.05): SPTB versus HTERM ( $\mathrm{n}=3705)$, SPTB versus PPROM $(n=70)$ and PPROM versus HTERM $(n=8469)$. However, the lowest $p$-value was determined for the gene set GSE37533 PPARG1-FOXP3-VS-FOXP3-TRANSDUCED-CD4-TCELL-DN (an 'immunologic signature') from the SPTB versus HTERM analysis, whereby 57 input genes overlapped (FDR p = 1.57E-13) (see Supplementary File 3). Peroxisome proliferator-activated receptor, or PPAR-gamma, are nuclear receptors involved with regulatory T cells, and FOXP3 (forkhead box P3) is part of a transcriptional factor family.

Comparison of SPTB versus HTERM DEGs to Genotype-Tissue Expression (GTEx) database determined a gene set (including 332 genes) that was significantly upregulated in whole blood at week 20 of gestation (FDR $\mathrm{p}=0.021$ ) (Supplementary Fig. S3, see Supplementary File 4). This included: TNFRSF4, TNFRSF1B, IL1B, IL1RN, $S L C 11 A 1, H L A-V, H L A-G$ and MIR142. Gene sets were also enriched in cervix/endocervix, ovary and vagina (but not significant at FDR $\mathrm{p}<0.05$ ).

EQTL mapping at week 16 of gestation. From the GWAS analyses of each phenotype, a total of 876 SNPs reached suggestive threshold $\left(\mathrm{p}<1 \times 10^{-5}\right)$ and were included for eQTL mapping. Of all three phenotype analyses, only the SPTB versus LTERM eQTL mapping resulted in 90 significant cis-eQTL transcript-SNP hits $(\mathrm{p}<0.05)$ (Supplementary Fig. S4). One of these significant transcript-SNP pair, TC1100011170.hg.1-rs76196041, was significant at FDR $\mathrm{p}<0.05$ (FDR $\mathrm{p}=0.019)$. Both the SNP and transcript were located in non-coding regions of chromosome 11 (see Supplementary File 5). Significant microRNA transcripts $(p<0.05)$ detected included microRNA 548L, microRNA 1343 and microRNA 139. A total of 219,593 trans-eQTL were highlighted for SPTB versus LTERM, of which 7 were significant at FDR $p<0.05$, all of which were in either unknown regions or non-coding regions. Several trans-eQTLs were also significant at FDR $\mathrm{p}<0.05$ for the remaining phenotype comparisons: PPROM versus HTERM $(n=23)$, SPTB versus HTERM $(n=72)$, PPROM versus LTERM $(n=23)$ (see Supplementary File 6).

EQTL mapping at week 20 of gestation. At week 20 of gestation only SPTB versus LTERM yielded significant cis-eQTLs. At $\mathrm{p}<0.05,91$ significant cis-eQTLs were detected, however none of the were significant after FDR p $<0.05$ (Supplementary Fig. S5). Of the 217,045 significant trans-eQTLs $(\mathrm{p}<0.05)$, 43 were significant at FDR $\mathrm{p}<0.05$.

SPTB versus HTERM trans-eQTL mapping at week 20 of gestation highlighted one significant coding transcript TC1400008661.hg.1 (TRDV3, T cell receptor delta variable 3 on chromosome 14) in association with SNP chr1.177008414_C (rs146756455, an intron variant of ASTN1, Astrotactin 1, on chromosome 1) (FDR $\mathrm{p}=0.04883$ ). The remaining transcripts and SNPs were identified in non-coding regions. Trans-eQTLs that reached FDR $\mathrm{p}<0.05$ for the remaining phenotypes included: PPROM versus HTERM $(\mathrm{n}=10)$, SPTB versus HTERM $(n=65)$, PPROM versus LTERM $(n=62)$ (see Supplementary File 6).

\section{Discussion}

The findings of this study strongly indicate different molecular attributes of PPROM and SPTB when compared with the term phenotypes, HTERM (women with a history of preterm birth and subsequent natural term birth) and LTERM (women with successive term births).

Two genome-wide significant SNPs were detected, ASTN1 (rs146756455, SPTB versus HTERM) (Fig. 3c) and MAST1 (rs188343966, sPTB cases versus LTERM) (Fig. 4b). Both genes are associated with neurodevelopment disorder in preterm infants ${ }^{31,32}$. ASTN1 was associated with prenatal development by Lionel et al. ${ }^{31}$, whereas MAST1 was reported in preterm infants, after birth, by Arpón et al. ${ }^{32}$. This correlates to our findings that (1) when all sPTB cases genotypes were compared to LTERM healthy pregnancies MAST1 was detected, (2) when the pregnancy outcomes were further stratified only SPTB genotypes were associated with ASTN1 when compared with term pregnancies that did not have a recurrent SPTB (HTERM). This suggests that ASTN1 is a candidate marker for the SPTB phenotype. These results also imply that perturbations in the process of neurodevelopment via various molecular pathways, which would lead to poor prognosis in preterm infants, could in fact be detected in early stages of pregnancy. Furthermore, it suggests a genetic difference between women who delivered term in all pregnancies (LTERM) and those who delivered sPTB followed by a term delivery (HTERM). This finding is novel in the obstetrics field of research. In terms of clinical management of patients, a genetic biomarker could aid risk stratification of high-risk women in a subsequent pregnancy.

The transcriptomics results suggest that local inflammation, potentially induced by TNF, occur in women who experienced PPROM, which is concordant with Capece et al. ${ }^{18}$ findings. The results further imply that RNA from maternal blood sampled at week 20 gestation could be utilised for predicting risk of early labour. Gene expression analysis highlighted microRNA 142 as significant in the PPROM versus HTERM outcome comparison at week 20 of gestation (FDR p=0.02) (Table 1, Fig. 5). A PTB study of cervical cells by Sanders et al..$^{23}$ reported 
increased expression of microRNA 142 (and 5 other microRNAs) in preterm cases compared to term controls, thereby associating this transcript with shorter gestational length. Sanders et al. ${ }^{23}$ conducted network analysis of mRNA targets of the 6 upregulated microRNA (including microRNA 142) to identify molecules involved in DNA replication and inflammatory processes. This included a key role of tumour necrosis factor (TNF), which can induce inflammation resulting in preterm delivery ${ }^{33,34}$. IL1RN was also detected (Table 1) and is related to early onset of labour due to infection ${ }^{35-40}$.

Sanders et al. ${ }^{23}$ also determined DNA methyltransferases in the network analysis and DNA methylation is known to supress microRNA 142, which suggests a role of epigenetics in $\mathrm{PPROM}^{41}$. Future work would include collecting and investigating epigenetic data from this cohort of women and comparing back to these findings for further insight on the potential mechanism of microRNAs and inflammatory response in PPROM.

The role of inflammation and immune response is also likely for women experiencing SPTB, though the different molecular signatures suggest that different mechanisms are activated compared to PPROM. SPTB versus HTERM (at week 20 of gestation) gene set enrichment analysis highlighted the significant gene set GSE37533, an "immunologic signature". This gene set consists of PPAR-gamma, which initiates adipocyte differentiation and has been suggested to combine with Foxp3 to regulate transcriptional signatures of regulatory T-cells ${ }^{42}$. In addition to this immunological finding, a significantly upregulated gene set in whole blood including many inflammation-associated genes was identified (Supplementary Fig. S3). Detection of such inflammation biomarkers in the blood could lead to the development of a non-invasive screening tool for clinical practice. Cis-eQTL mapping highlighted one significant transcript-gene pair (FDR p <0.05) at week 16 of gestation. No biological plausibility was identified for rs76196041 or the transcript as they were both located in non-coding regions. Rs76196041 detected in the GWAS analysis of SPTB versus LTERM $(p=8.15 \mathrm{e}-06)$ did not meet genome-wide significance but reached above the arbitrary suggestive threshold of $\mathrm{p}<1 \times 10^{-5}$ (Fig. 3 ). This implies that the association of rs76196041 between SPTB and LTERM could distinguish between the two phenotypes. This is particularly of importance as the LTERM group were healthy controls who never experienced sPTB. Through many trans-eQTLs were significant at both timepoint analyses, gene expression is more likely to be affected by SNPs that are closer to the gene loci therefore cis-eQTLs are of interest ${ }^{43,44}$.

One of the major strengths of the study include the unique study cohort. Women were prospectively recruited in Liverpool and applied a threshold of $\leq 34$ weeks of gestation to capture clinically well-defined sPTB pregnancy outcomes. In addition to this, two control groups, LTERM healthy controls and HTERM women who delivered term after a previous SPTB. Information on recurrent pregnancies does not exist in other biobanks. Another key strength was that both genomic and transcriptomic data were obtained from the same women (at two timepoints), and this was investigated using unbiased genome and transcriptome profiling technologies to detect molecular signatures. This provided an insight into the interactions between the omic layers and determine potential mechanistic pathways, which is a benefit of multi-omic studies.

Due to the clear definition of each SPTB group, the numbers of cases were low in this study. Therefore, differences in gene expression between PPROM and SPTB could not be detected. However, when these cases were compared with term births, molecular signatures were detected, indicating that there are genetic differences between SPTB and PPROM when compared to term birth outcomes. This limitation was also present for the GWAS analyses; however, the detection of genome-wide significant SNPs warrants further investigation for instance with PCR methods. Furthermore, LTERM samples could not be processed on the Clariom D array, due to limited funds. An advantage of eQTL mapping was that it allowed for SNPs from LTERM GWAS analyses to be correlated to expression data of HTERM or sPTB births.

\section{Conclusion}

This study identified multiple inflammation biomarkers of sPTB in a unique, well-defined cohort of women who delivered $\leq 34$ weeks of gestation. This study has also demonstrated the potential for multi-omics biomarkers as a diagnostic tool to detect if a woman is at risk of delivering preterm and thereby enabling early clinical intervention. Future work involves validation of the genes and transcripts identified in our cohort samples. Integration of this data with other omics, such as metabolomics would direct this analysis towards identification of potential pathways involved with the initiation of PTB.

\section{Methods}

Study participants. Women were recruited at 16 and 20 weeks of gestation in a subsequent singleton pregnancy at the Liverpool Women's Hospital Preterm Birth Prevention Clinic, between April 2012 and December 2017. An additional singleton 'low risk for PTB' pregnancy population with a history of only term births ( $\geq 39$ weeks of gestation) were also recruited at 16 and 20 weeks. Participants were included in the study if they were $>18$ years old, willing to undergo transvaginal ultrasound scan and were able to provide written consent. For women 'high-risk of PTB', a further inclusion criterion of previous PPROM or SPTB ( $>16$ and $\leq 34$ weeks of gestation). The exclusion criteria are reported in Fig. 1.

Study sample size and power calculations were conducted based on data obtained from the recruitment of a pilot cohort of women. Further details are provided in Supplementary Methods and Supplementary Fig. S6 (see Supplementary File 1). All pregnancies were followed up and clinical data were collected. Informed consent was obtained from participants. Research ethics approval for this nested case-control study was obtained from the North West Liverpool Central Research Ethics Committee (REC reference: 11/NW/0720). This study was conducted in accordance with institutional and national ethical standards and complied with the 1964 Helsinki Declaration and its later amendments or comparable ethical standards.

Participants were categorised into mutually exclusive phenotypes based on their current pregnancy outcome. For women considered high risk of PTB (history of PTB $<34$ weeks): HTERM (birth $\geq 37^{+0}$ weeks) or SPTB 
(spontaneous preterm birth) or PPROM (preterm premature rupture of the membranes with $>12 \mathrm{~h}$ prior to labour onset) (PTB $\leq 34^{+0}$ weeks gestation). Low-risk women with a recurrent term pregnancy $\left(\geq 39^{+0}\right.$ weeks), were our control group, labelled LTERM and represented normality.

Preterm cases, SPTB and PPROM, were combined as a "PTB cases $<34$ week" group and compared against both high risk (HTERM) and low risk controls (LTERM) to determine if statistical power in our GWAS was increased.

Sample preparation, quality checks and microarrays. Whole blood samples were collected in BD vacutainer $\mathrm{K}_{2}$ EDTA tubes. DNA was extracted using the Chemagenic Magnetic Separation Module I (Auto Q Biosciences Ltd, UK) from maternal whole blood $(\mathrm{N}=369)$. Oxford Genomics Centre at the Wellcome Centre for Human Genetics processed the DNA samples on the Applied Biosystems ${ }^{\mathrm{TN}}$ UK Biobank Axiom ${ }^{\mathrm{Tn}}$ array (Thermo Fisher Scientific) for genome-wide screening.

Further whole blood samples were collected in PAXgene Blood RNA Tubes (PreAnalytiX, QIAGEN) and stored at $-80^{\circ} \mathrm{C}$. Total RNA $(\mathrm{N}=115)$ was extracted using spin column PreAnalytix kit (PreAnalytiX, QIAGEN). Sample quality was measured using the RNA 6000 Nano and Pico Kit and Agilent 2100 BioAnalyzer (Agilent Technologies). Samples with RNA integrity number (RIN) of $>7$ were hybridised to the GeneChip ${ }^{\text {Tx }}$ Clariom $^{\text {Thx }} \mathrm{D}$ Human assay (Thermo Fisher Scientific).

Data generated from the array platforms have been deposited at the European Genome-phenome Archive $(E G A)^{45}$, which is hosted by the EBI and the CRG, under the study accession number EGAS00001005076.

GWAS data quality control. Genotyping with the Axiom ${ }^{\mathrm{ma}}$ array was performed and Axiom ${ }^{\mathrm{Tm}}$ Analysis Suite 2.0 was applied to identify samples that passed $97 \%$ genotype call rate by Oxford Centre for Genomics (Wellcome Centre for Human Genetics, University of Oxford). GWAS standard quality control procedures reported by Anderson et al. ${ }^{46}$ and Marees et al. ${ }^{47}$ were followed using PLINK v1.9 software ${ }^{48}$.

Samples were excluded due to the following reasons: overall heterozygosity rate was \pm 3 standard deviations from the cohort mean a high proportion of missing SNPs; heterozygosity on chromsome X resulting in gender discrepancy or indication of close relatedness (or 'identical by descent', IBD) with other samples. Samples were included if genetically assigned to European ancestry (CEU) population based on the HapMap data ${ }^{49,50}$ (Supplementary Fig. S7).

SNPs were excluded if they had a low genotype call rate $(<95 \%)$; minor allele frequency (MAF) of $<1 \%$ and if SNPs deviated from Hardy-Weinberg Equilibrium (HWE) at $\mathrm{p} \leq 1 \times 10^{-647}$. Prior to imputation, HRC Perl script v4.2.7 by Will Rayner was executed to remove trialleic, biallelic and palindromic SNPs.

Phasing and imputation of 618,283 SNPs was completed using the Michigan Imputation Server, applying Eagle v2.3 to phase chromosome 1 to 22 and minimac3 algorithm with the HRC r1.1 2016 reference panel for imputation ${ }^{51,52}$. Variants with $\mathrm{R}^{2}<0.3^{53}$ and $\mathrm{MAF}=0($ or $<1 \%)$ were excluded post-imputation to retain higher quality imputated SNPs.

GWAS analysis. Frequentist association analysis between the four individual phenotypes was completed using SNPTEST v2.5 $5^{5-56}$. Multi-dimensional scaling components 1 to 6 were included as covariates to control for genetic variance observed in the cohort. Manhattan plots were generated using R package 'qqman' ${ }^{28}$. Biomart Ensembl GRCh37 (release 97, EMBL-EBI), was applied for SNP annotation (SNPs reaching p $<1 \times 10^{-5}$ and genome-wide significance of $\left.\mathrm{p}<5 \times 10^{-8}\right)^{57}$. Regional plots were generated using LocusZoom (University of Michigan) web tool to explore SNPs in linkage disequilibrium ${ }^{29}$.

Differential gene expression analysis. Array data was pre-processed by performing Robust Multichip/ multi-array Analysis (RMA) using the R Bioconductor package, 'oligo ${ }^{58}$. Annotation was completed with $\mathrm{R}$ package 'affycoretools'59 and the Clariom D Human array database 'pd.clariom.d.human' 60 . Differential gene expression analysis was conducted on SPTB, PPROM and HTERM outcome comparisons (at both week 16 and 20 of gestation) using R package 'limma' with ANOVA and empirical Bayes ${ }^{30}$.

Pathway analysis. Gene symbols of significant DEGs (FDR $\mathrm{p}<0.05)$ from PPROM cases versus HTERM controls gene expression analysis were uploaded onto Reactome ${ }^{61,62}$ for pathway analysis. Missing gene symbols were excluded.

Gene set enrichment. FUMA GWAS online software (Functional Mapping and Annotation of GenomeWide Association Studies) was applied for gene set enrichment analysis (GSEA, Broad Institute, US) of genes identified from expression analysis across all phenotypes (using $\mathrm{p}<0.05$ threshold) ${ }^{63}$. Genotype-Tissue Expression (GTEx) database was used to identify whether these transcripts could impact tissue-specific gene expression.

Matrix eQTL analysis. R package 'MatrixEQTL'43 was applied for eQTL mapping of the gene expression data and SNPs with $\mathrm{p}<1 \times 10^{-5}$ from GWAS analyses comparing PPROM versus HTERM, PPROM versus LTERM, SPTB versus HTERM, SPTB versus LTERM. Linear regression model was applied, with thresholds of $\mathrm{p}<0.02$ for cis-eQTLs (local) and $\mathrm{p}<0.01$ for trans-eQTLs (distant). The test statistics for every transcript-SNP pair exceeding the threshold were returned with the corresponding $\mathrm{p}$-values. 
Ethics approval. Research ethics approval was Granted by the North West Research Ethics Committee (REC reference: 11/NW/0720).

Consent to participate. All participants provided informed consent for this study.

\section{Data availability}

The genomic and transcriptomic datasets generated and/or analysed during the current study are available in the European Genome-Phenome Archive (EGA) EBI repository (https://ega-archive.org/studies/EGAS000010 05076).

Received: 11 May 2021; Accepted: 23 December 2021

Published online: 19 January 2022

\section{References}

1. Johnson, S. \& Marlow, N. Early and long-term outcome of infants born extremely preterm. Arch. Dis. Child. 102, 97-102 (2017).

2. Luu, T. M., Rehman Mian, M. O. \& Nuyt, A. M. Long-term impact of preterm birth: Neurodevelopmental and physical health outcomes. Clin. Perinatol. 44, 305-314 (2017).

3. NHS England.Saving Babies' Lives Care Bundle Version 2. England.nhs.uk. https://www.england.nhs.uk/publication/saving-babieslives-version-two-a-care-bundle-for-reducing-perinatalmortality/ (2019).

4. Adams, M. M., Elam-Evans, L. D., Wilson, H. G. \& Gilbertz, D. A. Rates of and factors associated with recurrence of preterm delivery. JAMA 283, 1591-1596 (2000).

5. Bhattacharya, S. et al. Inherited predisposition to spontaneous preterm delivery. Obstet. Gynecol. 115, 1125-1133 (2010).

6. Mercer, B. M. et al. The preterm prediction study: effect of gestational age and cause of preterm birth on subsequent obstetric outcome. National Institute of Child Health and Human Development Maternal-Fetal Medicine Units Network. Am. J. Obstet. Gynecol. 181, 1216-1221 (1999).

7. Porter, T. F., Fraser, A. M., Hunter, C. Y., Ward, R. H. \& Varner, M. W. The risk of preterm birth across generations. Obstet. Gynecol. 90, 63-67 (1997).

8. Menon, R. Spontaneous preterm birth, a clinical dilemma: Etiologic, pathophysiologic and genetic heterogeneities and racial disparity. Acta Obstet. Gynecol. Scand. 87, 590-600 (2008).

9. Steffen, K. M. et al. Maternal and fetal variation in genes of cholesterol metabolism is associated with preterm delivery. J. Perinatol. 27, 672-680 (2007).

10. Frey, H. A. et al. Genetic variation associated with preterm birth in African-American women. Am. J. Obstet. Gynecol. 215, e1-e8 (2016).

11. Langmia, I. M., Apalasamy, Y. D., Omar, S. Z. \& Mohamed, Z. Interleukin 1 receptor type 2 gene polymorphism is associated with reduced risk of preterm birth. J. Matern Fetal Neonatal Med. 29, 3347-3350 (2016).

12. Menon, R. et al. Genetic regulation of amniotic fluid TNF-alpha and soluble TNF receptor concentrations affected by race and preterm birth. Hum. Genet. 124, 243-253 (2008).

13. Menon, R. et al. Multilocus interactions at maternal tumor necrosis factor-alpha, tumor necrosis factor receptors, interleukin-6 and interleukin-6 receptor genes predict spontaneous preterm labor in European-American women. Am. J. Obstet. Gynecol. 194, 1616-1624 (2006).

14. Ramos, B. R. et al. Ancestry informative markers and selected single nucleotide polymorphisms in immunoregulatory genes on preterm labor and preterm premature rupture of membranes: A case control study. BMC Pregn. Childbirth 16, 30 (2016).

15. Zhang, G. et al. Genetic associations with gestational duration and spontaneous preterm birth. N. Engl. J. Med. 377, 1156-1167 (2017).

16. Goldenberg, R. L., Culhane, J. F., Iams, J. D. \& Romero, R. Epidemiology and causes of preterm birth. Lancet 371, 75-84 (2008).

17. Romero, R., Dey, S. K. \& Fisher, S. J. Preterm labor: One syndrome, many causes. Science 345, 760-765 (2014).

18. Capece, A., Vasieva, O., Meher, S., Alfirevic, Z. \& Alfirevic, A. Pathway analysis of genetic factors associated with spontaneous preterm birth and pre-labor preterm rupture of membranes. PLoS ONE 9, el08578 (2014).

19. Care, A. et al. Should phenotype of previous preterm birth influence management of women with short cervix in subsequent pregnancy? Comparison of vaginal progesterone and Arabin pessary. Ultrasound Obstet. Gynecol. 53, 529-534 (2019).

20. Cook, J. et al. First trimester circulating microRNA biomarkers predictive of subsequent preterm delivery and cervical shortening. Sci. Rep. 9, 5861 (2019).

21. Elovitz, M. A. et al. Distinct cervical microRNA profiles are present in women destined to have a preterm birth. Am. J. Obstet. Gynecol. 210(221), e1-e11 (2014).

22. Gray, C., McCowan, L. M., Patel, R., Taylor, R. S. \& Vickers, M. H. Maternal plasma miRNAs as biomarkers during mid-pregnancy to predict later spontaneous preterm birth: A pilot study. Sci. Rep. 7, 815 (2017).

23. Sanders, A. P. et al. MicroRNA expression in the cervix during pregnancy is associated with length of gestation. Epigenetics 10, 221-228 (2015).

24. Zhou, G., Holzman, C., Heng, Y. J., Kibschull, M. \& Lye, S. J. Maternal blood EBF1-based microRNA transcripts as biomarkers for detecting risk of spontaneous preterm birth: A nested case-control study. J. Matern Fetal Neonatal Med. https://doi.org/10.1080/ 14767058.2020.1745178 (2020).

25. Cannell, I. G., Kong, Y. W. \& Bushell, M. How do microRNAs regulate gene expression? Biochem. Soc. Trans. 36, 1224-1231 (2008).

26. He, L. \& Hannon, G. J. MicroRNAs: Small RNAs with a big role in gene regulation. Nat. Rev. Genet. 5, 522-531 (2004).

27. Lawrenson, K. et al. Cis-eQTL analysis and functional validation of candidate susceptibility genes for high-grade serous ovarian cancer. Nat. Commun. 6, 8234 (2015).

28. Turner, S. D. Qqman: An R package for visualizing GWAS results using Q-Q and manhattan plots. J. Open Source Softw. 3, 731 (2018)

29. Pruim, R. J. et al. LocusZoom: Regional visualization of genome-wide association scan results. Bioinformatics 26, 2336-2337 (2010).

30. Ritchie, M. E. et al. limma powers differential expression analyses for RNA-sequencing and microarray studies. Nucleic Acids Res. 43, e47 (2015).

31. Lionel, A. C. et al. Disruption of the ASTN2/TRIM32 locus at 9q33.1 is a risk factor in males for autism spectrum disorders, ADHD and other neurodevelopmental phenotypes. Hum. Mol. Genet. 23, 2752-2768 (2014).

32. Arpón, A. et al. Methylation changes and pathways affected in preterm birth: A role for SLC6A3 in neurodevelopment. Epigenomics 10, 91-103 (2018).

33. Gomez-Lopez, N., StLouis, D., Lehr, M. A., Sanchez-Rodriguez, E. N. \& Arenas-Hernandez, M. Immune cells in term and preterm labor. Cell Mol. Immunol. 11, 571-581 (2014).

34. Romero, R. et al. Tumor necrosis factor in preterm and term labor. Am. J. Obstet. Gynecol. 166, 1576-1587 (1992). 
35. Belousova, V. S., Svitich, O. A., Timokhina, E. V., Strizhakov, A. N. \& Bogomazova, I. M. Polymorphism of the IL-1 $\beta$, TNF, IL-1RA and IL-4 cytokine genes significantly increases the risk of preterm birth. Biochemistry (Mosc.) 84, 1040-1046 (2019).

36. Bitner, A. \& Kalinka, J. IL-1 $\beta$, IL-6 promoter, TNF- $\alpha$ promoter and IL-1RA gene polymorphisms and the risk of preterm delivery due to preterm premature rupture of membranes in a population of Polish women. Arch. Med. Sci. 6, 552-557 (2010).

37. Chaves, J. H., Babayan, A., Bezerra Cde, M., Linhares, I. M. \& Witkin, S. S. Maternal and neonatal interleukin-1 receptor antagonist genotype and pregnancy outcome in a population with a high rate of pre-term birth. Am. J. Reprod. Immunol. 60, 312-317 (2008).

38. Gillespie, S. L. et al. Interleukin-1 receptor antagonist polymorphism and birth timing: Pathway analysis among African American women. Nurs. Res. 66, 95-104 (2017).

39. Murtha, A. P. et al. Association of maternal IL-1 receptor antagonist intron 2 gene polymorphism and preterm birth. Am. J. Obstet. Gynecol. 195, 1249-1253 (2006).

40. Nadeau-Vallée, M. et al. Novel noncompetitive IL-1 receptor-biased ligand prevents infection- and inflammation-induced preterm birth. J. Immunol. 195, 3402-3415 (2015).

41. Skarn, M., Baroy, T., Stratford, E. W. \& Myklebost, O. Epigenetic regulation and functional characterization of microRNA-142 in mesenchymal cells. PLoS ONE 8, e79231 (2013).

42. Cipolletta, D. et al. PPAR- $\gamma$ is a major driver of the accumulation and phenotype of adipose tissue Treg cells. Nature 486, 549-553 (2012).

43. Shabalin, A. A. Matrix eQTL: Ultra fast eQTL analysis via large matrix operations. Bioinformatics 28, 1353-1358 (2012).

44. Wright, F. A., Shabalin, A. A. \& Rusyn, I. Computational tools for discovery and interpretation of expression quantitative trait loci. Pharmacogenomics 13, 343-352 (2012).

45. Lappalainen, I. et al. The European Genome-phenome archive of human data consented for biomedical research. Nat. Genet. 47, 692-695 (2015).

46. Anderson, C. A. et al. Data quality control in genetic case-control association studies. Nat. Protoc. 5, 1564-1573 (2010).

47. Marees, A. T. et al. A tutorial on conducting genome-wide association studies: Quality control and statistical analysis. Int. J. Methods Psychiatr. Res. 27, e1608 (2018).

48. Chang, C. C. et al. Second-generation PLINK: Rising to the challenge of larger and richer datasets. Gigascience 4, 7 (2015).

49. International HapMap Consortium. The International HapMap Project. Nature 426, 789-796 (2003).

50. International HapMap Consortium. A haplotype map of the human genome. Nature 437, 1299-1320 (2005).

51. Das, S. et al. Next-generation genotype imputation service and methods. Nat. Genet. 48, 1284-1287 (2016).

52. Loh, P. R. et al. Reference-based phasing using the haplotype reference consortium panel. Nat. Genet. 48, 1443-1448 (2016).

53. Schurz, H. et al. Evaluating the accuracy of imputation methods in a five-way admixed population. Front. Genet. 10, 34 (2019).

54. Marchini, J., Howie, B., Myers, S., McVean, G. \& Donnelly, P. A new multipoint method for genome-wide association studies by imputation of genotypes. Nat. Genet. 39, 906-913 (2007).

55. Marchini, J. \& Howie, B. Genotype imputation for genome-wide association studies. Nat. Rev. Genet. 11, 499-511 (2010).

56. Wellcome Trust Case Control Consortium. Genome-wide association study of 14,000 cases of seven common diseases and 3,000 shared controls. Nature 447, 661-678 (2007).

57. Smedley, D. et al. BioMart-Biological queries made easy. BMC Genomics 10, 22 (2009).

58. Carvalho, B. S. \& Irizarry, R. A. A framework for oligonucleotide microarray preprocessing. Bioinformatics 26, 2363-2367 (2010).

59. MacDonald, J. W. Affycoretools: Functions Useful for Those Doing Repetitive Analyses with Affymetrix GeneChips. R Package Version 1.58.4 (2020)

60. MacDonald, J.pd.clariom.d.human: Platform Design Info for Affymetrix Clariom_D_Human. R Package Version 3.14.1 (2016).

61. Fabregat, A. et al. The reactome pathway knowledgebase. Nucleic Acids Res. 46(D1), D649-D655 (2018).

62. Fabregat, A. et al. Reactome pathway analysis: A high-performance in-memory approach. BMC Bioinform. 18, 142 (2017).

63. Watanabe, K., Taskesen, E., van Bochoven, A. \& Posthuma, D. Functional mapping and annotation of genetic associations with FUMA. Nat. Commun. 8, 1826 (2017).

\section{Acknowledgements}

We would like to acknowledge the Centre for Genomic Research, University of Liverpool for generating the gene expression data (Clariom ${ }^{\text {Tx }}$ D Human assay) and the Oxford Genomics Centre at the Wellcome Centre for Human Genetics (funded by Wellcome Trust grant reference 090532/Z/09/Z) for acquiring the genotype data (UK Biobank Axiom ${ }^{\text {тx }}$ array). We would also like to thank our funders, Wellbeing of Women (UK).

\section{Author contributions}

Z.A., A.C. and L.G. (Obstetrics and Gynaecology clinicians) recruited the study participants. Data analyses were completed by J.G. and B.M.M. J.G. wrote the manuscript. All authors participated in the study design and prepared the manuscript.

\section{Funding}

The Harris-Wellbeing Research Centre was funded by the Wellbeing of Women charity, London, for this research.

\section{Competing interests}

The authors declare no competing interests.

\section{Additional information}

Supplementary Information The online version contains supplementary material available at https://doi.org/ 10.1038/s41598-022-04881-0.

Correspondence and requests for materials should be addressed to J.K.G.

Reprints and permissions information is available at www.nature.com/reprints.

Publisher's note Springer Nature remains neutral with regard to jurisdictional claims in published maps and institutional affiliations. 
(c) (i) Open Access This article is licensed under a Creative Commons Attribution 4.0 International cc) License, which permits use, sharing, adaptation, distribution and reproduction in any medium or format, as long as you give appropriate credit to the original author(s) and the source, provide a link to the Creative Commons licence, and indicate if changes were made. The images or other third party material in this article are included in the article's Creative Commons licence, unless indicated otherwise in a credit line to the material. If material is not included in the article's Creative Commons licence and your intended use is not permitted by statutory regulation or exceeds the permitted use, you will need to obtain permission directly from the copyright holder. To view a copy of this licence, visit http://creativecommons.org/licenses/by/4.0/.

(C) The Author(s) 2022, corrected publication 2022 